УДК 004.9: 629.78 DOI 10.30894/issn2409-0239.2020.7.1.51.58

\author{
Особенности формирования \\ информационно-вычислительной системы \\ для решения измерительных задач \\ в условиях различной степени структуризации \\ В. К. Ларин, к.m.н., c.н.c., contact@spacecorp.ru \\ АО «Российские космические системь», Москва, Российская Федерация
}

\begin{abstract}
Аннотация. В статье рассмотрены вопросы формирования информационно-вычислительной системы (ИВС) для решения измерительных задач в условиях различной степени структуризации. Решение задачи основано на принципах структурного анализа, центральным из которых является разделение сложной системы на простые составляющие с определением функциональных связей между ними. Вид решения может быть представлен как в графической, так и в аналитической формах. В работе сформулирована последовательность этапов решения поставленной задачи.

Приводится общая структурная схема ИВС для решения технических задач. В соответствии с указанными принципами решение разбивается на методическую и технологическую части. Даны структурные схемы этих частей. Выделен фрагмент решения измерительной задачи, отражающий специфику перехода решения от предыдущего блока к последующему в зависимости от выполнения «условий перехода». Показана зависимость «условий перехода» от значений информационных параметров. На примере задачи определения параметров движения КА рассмотрено применение сформированной ИВС для ее решения. Итогом исследований является структурная схема универсальной ИВС для решения технических задач различного типа.
\end{abstract}

Ключевые слова: информационно-вычислительная система, измерительная задача, структура, условия перехода, информационные параметры

\title{
Special Features of Building an Information Computer System for Solving Measuring Problems in Conditions of Varying Degrees of Structuring
}

\author{
V. K. Larin, Cand. Sci. (Engineering), senior researcher, contact@spacecorp.ru \\ Joint Stock Company "Russian Space Systems", Moscow, Russian Federation
}

\begin{abstract}
The article considers the creation of an information computer system (ICS) for solving measuring problems in conditions of different degrees of structuring. The solution is based on the principles of structural analysis. The key principle is the division of a complex system into simple constituents and the definition of functional relationships between them. The solution type can be given in both graphical and analytical form. The paper formulates a sequence of stages for solving the problem.

A general block diagram of the ICS for solving technical problems is given. In accordance with the aforementioned principles, the solution is divided into a methodological and a technological part. Block diagrams of these parts are presented. A fragment of the solution to the measuring problem, which reflects the particular nature of solution transition from the previous block to the next depending on the fulfillment of the "transition conditions", is highlighted. The dependence of the "transition conditions" on the values of information parameters is shown. The problem of determining the motion parameters of a spacecraft is considered as an example of implementing the developed ICS for its solution.
\end{abstract}

Keywords: information computer system, measuring task, structure, transition conditions, information parameters 


\section{Введение}

Объектом исследования является некоторая измерительная задача, в которой определены входные данные, вид результата и подход к решению данной задачи.

Измерительная задача - это задача обработки измерений с целью определения физических свойств (или характеристик) измеряемого объекта [1].

Примером может служить задача определения параметров движения КА, где объектом является орбита KA.

В основу исследований положены принципы структурного анализа [2].

Решение любой инженерной задачи можно разделить на две части: методическую, состоящую из постановки задачи и алгоритма, и технологическую, включающую программу, информационно-вычислительную систему (ИВС) и процедуру реализации программы на вычислительных средствах ИВС.

Согласно ГОСТ Р 53622-2009 термин информационно-вычислительная система означает совокупность данных (или баз данных), систем управления базами данных и прикладных программ, функционирующих на вычислительных средствах как единое целое для решения определенных задач [3].

Решение задачи зависит от степени ее структуризации. Согласно классификации, предложенной в [4], проблемы (к ним относятся и измерительные задачи) можно разделить по степени структуризации на три типа: структурированные (С3) имеющие численное решение; неструктурированные (НС3) - не имеющие решения; и слабоструктурированные (ССЗ), имеющие на фоне формаль- ного (численного) решения качественное решение, а в пределе - не имеющие решения.

\section{Решение задач различной степени структуризации}

На основе вышесказанного можно предположить, что слабоструктурированные задачи занимают пространство между структурированными и неструктурированными задачами. Очевидно, что степень структуризации задач на границе массива структурированных задач будет максимальной (примем равной 1) и минимальной - на границе массива неструктурированных задач (примем равной 0).

С большой долей вероятности можно предположить, что задачи со значением степени структуризации, близким к 1, должны иметь количественное или качественное решение, задачи со значением степени структуризации, близким к 0, должны иметь качественное решение, а в пределе - не иметь его.

Для достаточно большого диапазона, в который попадают ССЗ, и неопределенной корректности их решения, в [5] было предложено разделить массив слабоструктурированных задач на две части: задачи, примыкающие к массиву структурированных задач, названные квазиструктурированными задачами (KC3); и собственно слабоструктурированные задачи.

C учетом вышесказанного, а также приведенных выше значений степени структуризации (обозначим через K) массив КСЗ приближенно характеризуется диапазоном $\mathrm{K}$ от 1,0 до 0,5, массив СС3 соответственно диапазоном $\mathrm{K}$ от 0,5 до 0. В табл. 1 приведены в общем виде данные взаимосвязи вида решения задачи и степени структуризации.

Т абли ц а 1. Соответствие вида решения задачи и степени структуризации

\begin{tabular}{|c|c|c|c|}
\hline Типы задач & Степень структуризации $(\mathrm{K})$ & Методы решения & Примечание \\
\hline С3 & 1 & Аналитические методы & Численное решение \\
\hline КС3 & $1-0,5$ & Аналитические методы & $\begin{array}{c}\text { Численное решение с отклонением } \\
\text { точности результата }\end{array}$ \\
\hline СС3 & $0,5-0$ & Эвристические методы & Качественное решение \\
\hline НС3 & 0 & Нет решения & - \\
\hline
\end{tabular}


Практически все измерительные задачи изначально имеют численное решение. При реализации задачи на ИВС возможно получение результата с различной степенью точности. Это объясняется ошибками, допущенными при разработке алгоритма и программы, а также при формировании отдельных частей ИВС. Примем, что решение, полученное с ошибкой больше заданного предела, является непригодным для дальнейшего рассмотрения.

Таким образом, цель настоящих исследований заключается в формировании ИВС, которая позволяла бы получить оптимальный результат для измерительных задач квазиструктурированного типа в виде численного решения, так как качественное решение для измерительных задач не имеет практического значения.

В статье рассмотрена технология построения ИВС для решения данной проблемы.

Решение поставленной задачи заключается в последовательном выполнении следующих этапов.

Этап 1. Определение структуры ИВС. На первом этапе определяется структура ИВС, используемая для решения информационных задач общего типа. Состав такой ИВС определен в ГОСТ P 53622-2009.

Этап 2. Определение методической и технологической частей решения измерительных задач. На этом этапе определяется структура методической и технологической частей решения измерительных задач, проводится анализ структур с целью выделения блоков, несущих наибольшую нагрузку некорректности в элементах, и определяется влияние изменения характеристик элементов некорректных блоков на точность решения задачи в заданной предметной области.

Этап 3. Формирование основной структуры ИВС. На третьем этапе формируется основная структура ИВС с блокировкой решений, полученных с ошибками, превышающими предельные значения.

\section{Этап 1. Определение структуры ИВС}

В соответствии с требованиям ГОСТ Р 536222009 ИВС включает следующие виды обеспечения: информационное, программное, алгоритмическое и техническое. С учетом сказанного струк-

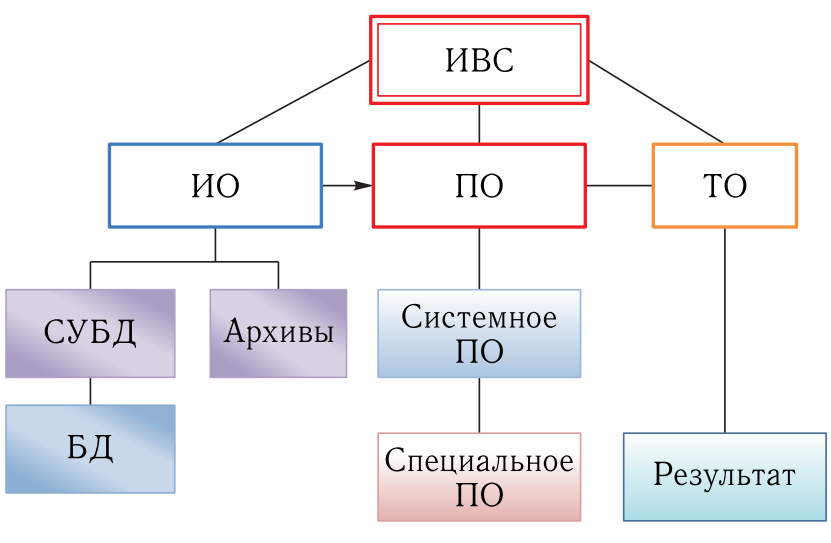

Рис. 1. Структурная схема ИВС

турная схема ИВС общего типа для решения технических задач в технологическом варианте будет иметь следующий вид (рис. 1).

На рис. 1 введены сокращения: ИО - информационное обеспечение, ПО - программное обеспечение, ТО - техническое обеспечение, БД - база данных, СУБД - система управления БД.

Алгоритмическое обеспечение не включено в схему, так как оно отнесено к методической части и рассматривается отдельно.

\section{Этап 2. Определение методической и технологической частей решения измерительных задач}

Специфика измерительных задач (ИЗ) характеризуется следующими свойствами:

- большим объемом входных данных (измерений), ошибки которых имеют в основном случайный характер;

- используемыми методами обработки - фильтрация измерений по критериям с последующей обработкой статистическими методами (метод наименьших квадратов, метод Монте-Карло);

- строгим соблюдением правил формирования и решения условных и нормальных уравнений поправок к измерениям (соответствие числа уравнений числу неизвестных);

- заданием оптимальных значений пределов внутренней сходимости в процедурах решения с использованием метода последовательных приближений (согласование быстродействия и точности решения). 
Указанные свойства измерительных задач значительно усложняют поиск решения. Во-первых, необходимостью формализации свойств для их учета и, во-вторых, определением возможного диапазона их значений. Для решения этих вопросов целесообразно использовать методы структурного анализа, а также подход, используемый в [6], где рекомендуется предварительно разделить процедуру решения на методическую и технологическую части, представив каждую часть в виде структурной схемы. Структурные схемы методической и технологической частей решения представлены на рис. 2 и 3 соответственно.

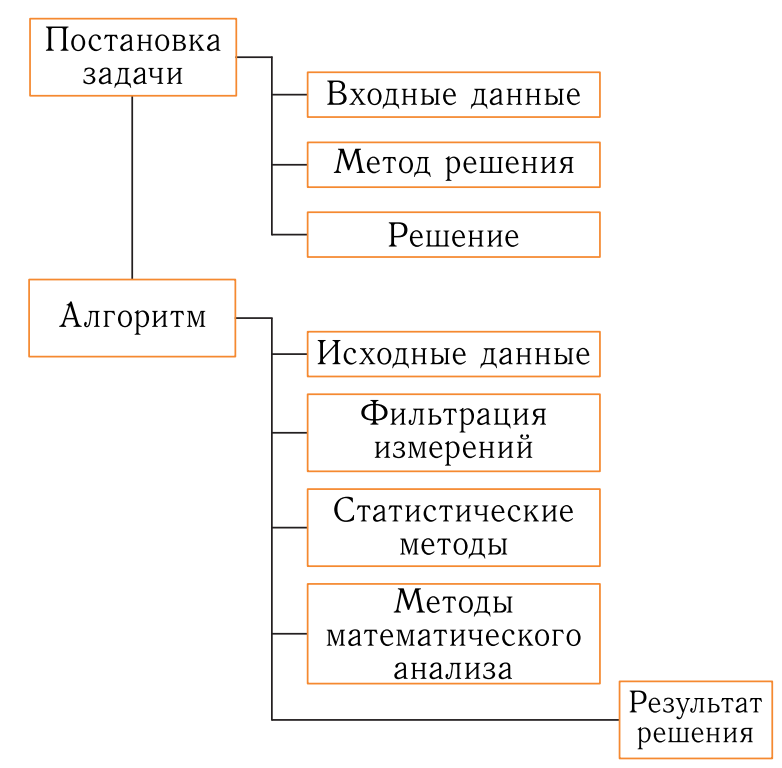

Рис. 2. Структура методической части решения

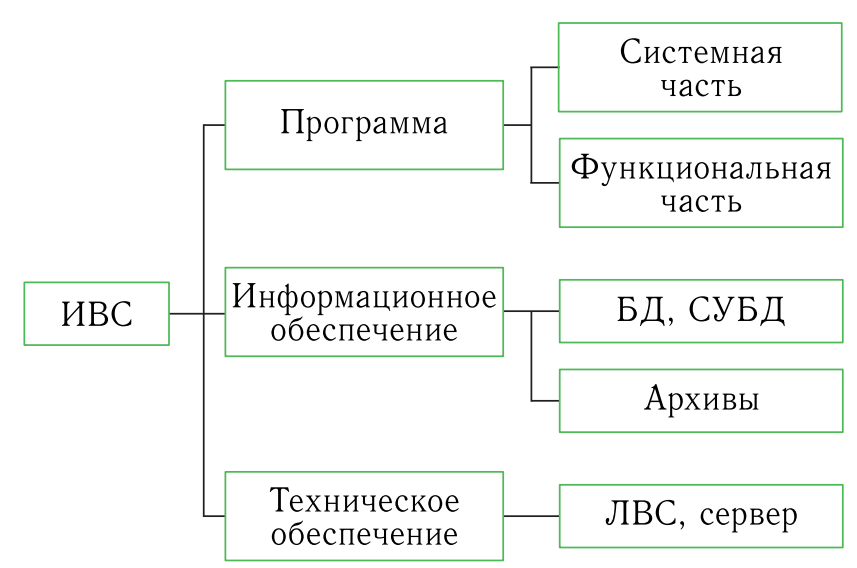

ЛВС - локальная вычислительная сеть

Рис. 3. Структура технологической части решения
Сам по себе термин «слабоструктурированная проблема (задача)» говорит о «слабых» структурных связях частей системы или об их отсутствии. Аналитически связи можно описать с помощью условий перехода между соседними блоками программы. По аналогии со структурными связями условия могут отсутствовать или быть не точно сформулированными. В таком случае либо может произойти сбой решения, либо получен отличающийся по точности результат.

Возможны следующие варианты «сбоя» на некотором шаге программы:

- остановка расчета вследствие невыполнения условия перехода к очередному блоку программы (команда «Останов»);

- получение неверных данных в очередном блоке программы вследствие влияния критического значения информационного параметра (ИП) и переход к следующему блоку программы на очередном шаге за счет изменения условий перехода.

Структурная схема фрагмента «сбойного» варианта решения при возможном нарушении условий перехода между соседними блоками программы представлен на рис. 4.

На рис. 4: $U\left(k_{n}\right)$ - номинальное условие перехода от блока $n$ к блоку $n+1 ; I_{n}-$ ИП блока $n$; $U\left(k_{n}, I_{n}\right)$ - измененное условие перехода от блока $n$ к блоку $n+1 ; N-$ номинальное значение

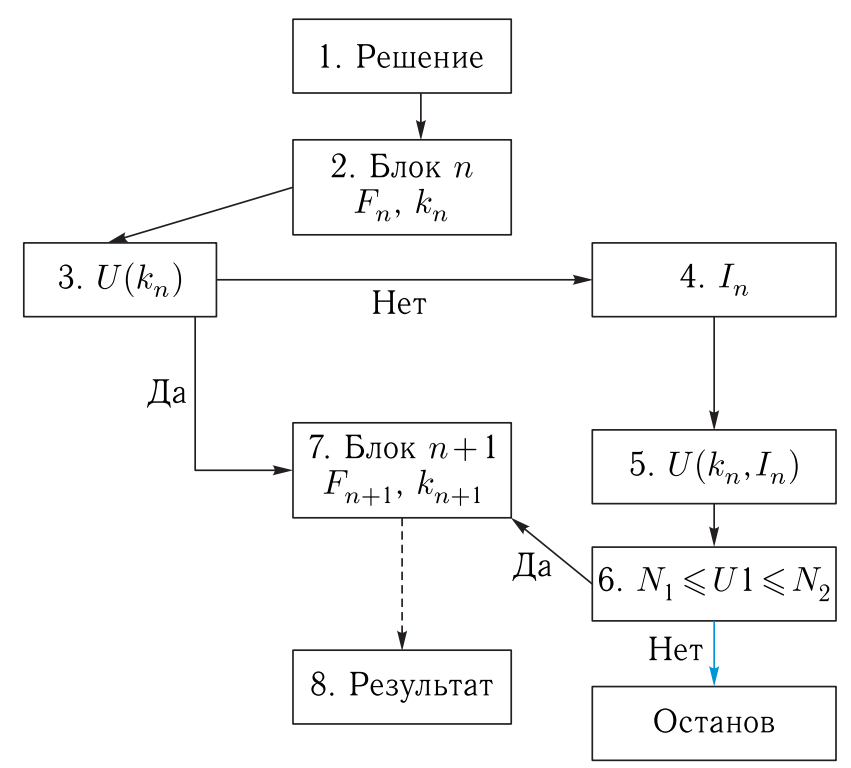

Рис. 4. Схема фрагмента перехода расчетов между соседними блоками программы $n, n+1$ 
условия перехода; $N_{1}-N_{2}-$ диапазон значений условия перехода для конкретного блока; $U 1=$ $=U\left(k_{n}, I_{n}\right)$ (в блоке 6$) ; F_{n}, F_{n+1}-$ функциональное содержание блоков $n, n+1$.

В алгоритмической форме этот фрагмент может быть представлен в следующем виде:

1. $F_{n}$ - расчет блока $n$.

2. $U\left(k_{n}\right)=N>F_{n+1} \neq \rightarrow I_{n}$.

3. $U\left(k_{n}, I_{n}\right)=U 1-$ коррекция условия перехода (УП) как функции информационного параметра.

4. $N_{1} \leqslant U 1 \leqslant N_{2} \rightarrow F_{n+1}, \geqslant \rightarrow$ Останов, где $U_{n}-$ номинал условия перехода блока $n$;

$I_{n}-$ информационный параметр блока $n$;

$k_{n}-$ критерий расчета $F_{n}$;

$k_{n+1}-$ критерий расчета $F_{n+1}$;

$\delta$ - поправка к УП.

Для анализа условий перехода представим программу в виде набора замкнутых математических процедур, где переход между ними осуществляется по выполнению условий.

Вид условия зависит от содержания очередной процедуры (блока).

Для измерительных задач возможны следующие условия перехода:

- полученные данные на очередном шаге должны быть больше, меньше или равны некоторому значению;

- полученные данные должны находиться в заданном диапазоне значений;

- единицы измерений полученных данных должны соответствовать единицам измерений величин, используемых на следующем шаге решения (проверка на идентичность единиц измерения должна проходить на каждом шаге программы).

В качестве примера рассмотрим задачу определения параметров движения космического аппарата (ОПД КА), условиями перехода в которой могут служить следующие соотношения рассчитываемых параметров:

- при формировании двойных разностей измерений фазы длина базовой линии должна находиться в заданном диапазоне;

- при фильтрации измерений (кода или фазы) значение сигнал/шум каждого измерения не должен быть меньше заданной величины;

- при фильтрации измерений (кода или фазы) по углу места, при котором производилось измере- ние, его значение не должно быть меньше заданного;

- при формировании сеанса количество измерений не должно быть меньше заданного;

- при формировании математической модели движения (ММД) КА учитываемые возмущающие ускорения должны обеспечивать заданную точность параметров орбиты KA;

- при формировании математической модели измерений (ММИ) учитываемые составляющие должны соответствовать заданному набору поправок;

- при оценке решения «краевой задачи» по данным определения местоположения отличия полученных значений координат от расчетных не должны превышать заданной величины.

В соответствии с [7] ИП зависят от значений функций и процедур, составляющих основу решения, и могут совпадать с условиями перехода и влиять на точность результата. Ниже представлен перечень основных ИП ОПД КА.

Как и в предыдущем случае, ИП целесообразно разделить по их влиянию на две части: методическую (постановка и алгоритм) и технологическую (информационное обеспечение и программа).

ИП элементов методической части решения и их критические значения

Постановка задачи

1. Цель решения (неоднозначно сформулирована).

Aлгоритм

1. Количество измерений в сеансе (меньше нормы).

2. Учет возмущающих сил в модели движения (недостаточно).

3. Учет поправок в модели измерений (недостаточно).

4. Критерии фильтрации измерений (не соответствуют нормам).

5. Диапазон базовых линий (задан неверно).

6. Величина разрядки измерений (задана неверно).

ИП элементов технологической части решения и их критические значения

Информационное обеспечение

1. Координаты измерительных станций (заданы неверно). 
Т а бл и ц а 2. Перечень ИП и их значения

\begin{tabular}{|c|c|c|c|c|}
\hline $\begin{array}{c}\text { № } \\
\text { п/п }\end{array}$ & Наименование & Символ & $\begin{array}{c}\text { Соотношение } \\
\text { текущего } \\
\text { и номинального } \\
\text { значений ИП }\end{array}$ & Примечание \\
\hline \multicolumn{5}{|c|}{ Методическая часть } \\
\hline 1 & Цель решения & $\mathrm{Zl}$ & $\neq 0$ & Неоднозначность \\
\hline 2 & Количество измерений в сеансе & $n_{i}$ & $n_{i}<n_{s}$ & Число измерений меньше заданного \\
\hline 3 & Возмущающие силы в ММД & $F_{v}$ & $F_{v} \neq f_{v}$ & Недостаток учета возмущающих сил \\
\hline 4 & Поправки в модель измерений & $\delta I_{z}$ & $\delta I_{z} \neq \Delta I_{z}$ & Недостаток учета поправок в ММИ \\
\hline 5 & Критерии фильтрации & $F_{j}$ & $F_{j} \neq F_{N}$ & Критерий не соответствует норме \\
\hline 6 & Диапазон базовых линий & $\Delta L_{b}$ & $\Delta L_{b} \neq N$ & Базовые линии вне диапазона \\
\hline 7 & Величина разрядки измерений & $\Delta t_{r}$ & $\Delta t_{r} \neq \Delta t_{N}$ & Разрядка не соответствует норме \\
\hline \multicolumn{5}{|c|}{ Технологическая часть } \\
\hline 8 & Измерительные станции & $B_{s}, L_{s}$ & $B_{s}, L_{s} \neq B_{n}, L_{n}$ & Координаты ИС заданы неверно \\
\hline 9 & $\begin{array}{l}\text { Выбор данных из таблиц } \\
\text { (по транзакции) }\end{array}$ & $\operatorname{Tr}$ & $\operatorname{Tr}_{i}=0$ & Откат транзакции \\
\hline 10 & Таблицы & $q_{i}$ & $q_{i} \neq q_{n}$ & Данные в ячейках имеют ошибки \\
\hline 11 & Настройки & $N_{i}$ & $N_{i} \neq N_{z}$ & $\begin{array}{l}\text { Настройки не равны заданному } \\
\text { значению }\end{array}$ \\
\hline 12 & Выбор исходных данных из архива & $I_{D}$ & $I_{D} \neq I_{z}$ & ИД с ошибками \\
\hline 13 & Цикл & $Z_{t}$ & $Z_{t} \neq Z_{n}$ & Параметры цикла заданы неверно \\
\hline 14 & Условие & $\mathrm{If}_{t}$ & $\mathrm{If}_{t} \neq \mathrm{If}_{n}$ & Условие задано неверно \\
\hline 15 & Стандартная программа & Sp & $\mathrm{Sp} \neq \mathrm{Sp}_{n}$ & $\begin{array}{l}\text { Несоответствие норме выбранной } \\
\text { стандартной программы }\end{array}$ \\
\hline 16 & Формирование результата & $R$ & $R \neq R_{n}$ & Формирование неверного результата \\
\hline
\end{tabular}

2. Формирование таблиц (с ошибками).

3. Выбор данных из таблиц (откат транзакций).

\section{Программа}

1. Настройки (с ошибками).

2. Выбор исходных данных из архивов (с ошибками).

3. Циклы (с ошибками).

4. Условия (с ошибками).

5. Выбор и использование стандартных подпрограмм (с ошибками).

6. Формирование результата (с ошибками).
В табл. 2 приведен перечень ИП и их значений, приводящих решение к одному из сбойных вариантов.

Как показывает опыт работы, два случая могут привести к невозможности получить решение:

- неопределенность цели решения, что соответствует ИП в постановке задачи;

- ошибки при настройке программы, что соответствует ИП в программе.

Все остальные случаи приводят к неточному результату.

Результаты отбора ИП второго случая, в соответствии с [8], представлены в табл. 3. 
Т а бл и ц а 3. Результаты отбора ИП

\begin{tabular}{|c|c|c|c|}
\hline $\begin{array}{l}\text { № } \\
\text { П/ח }\end{array}$ & Название ИП & Критическое условие ИП & Примечание \\
\hline \multicolumn{4}{|c|}{ Архив } \\
\hline 1 & Диапазон базовых линий & $\delta l_{i}<\Delta l$ & Базовые линии вне диапазона \\
\hline 2 & Координаты измерительных станций & $B_{s}, L_{s} \neq B_{n}, L_{n}$ & Координаты ИС заданы неверно \\
\hline 3 & Исходные данные из архивов & $I_{D} \neq I_{z}$ & ИД заданы неверно \\
\hline \multicolumn{4}{|c|}{ БД, СУБД } \\
\hline 4 & Заполнение таблиц БД & $q_{i} \neq q_{n}$ & Ошибки в ячейках \\
\hline 5 & Выбор данных из таблиц БД & $\operatorname{Tr}_{i}=0$ & Откат транзакций \\
\hline \multicolumn{4}{|c|}{ Программа } \\
\hline 6 & Возмущающие силы в ММД & $F_{v} \neq f_{v}$ & Недостаток учета возмущающих сил \\
\hline 7 & Поправки в модель измерений & $\delta I_{z} \neq \Delta I_{z}$ & Недостаток учета поправок \\
\hline 8 & Критерии фильтрации & $F_{j} \neq F_{N}$ & Критерий не соответствует норме \\
\hline 9 & Величина разрядки измерений & $\Delta t_{r} \neq \Delta t_{N}$ & Разрядка не соответствуют норме \\
\hline 10 & Циклы & $Z_{t} \neq Z_{n}$ & Параметры цикла заданы неверно \\
\hline 11 & Логические условия & $\mathrm{If}_{t} \neq \mathrm{If}_{n}$ & Условие задано неверно \\
\hline 12 & Стандартные подпрограммы & $\mathrm{Sp} \neq \mathrm{Sp}_{n}$ & $\begin{array}{l}\text { Несоответствие норме выбранной } \\
\text { стандартной подпрограммы }\end{array}$ \\
\hline
\end{tabular}

\section{Этап 3. Формирование основной структуры ИВС}

С учетом схемы фрагмента программы (рис. 4) и данных табл. 3 можно сформировать ИВС для измерительной задачи определения параметров движения KA, учитывающую степень ее структуризации. Структурная схема ИВС представлена на рис. 5.

ИПА 1, 2, 3 - информационные параметры блока «Архив»; ИПД 1, 2 - информационные параметры блока БД, СУБД; блок $1,2, \ldots, n-1, n-$ блоки программы; фрагмент $1,2, \ldots, n-1, n-$ подпрограмма перехода между блоками, индекс соответствует номеру блока (рис. 4 ); $\sigma_{r}-$ среднеквадратическая ошибка результата; $N_{3}$ - предельное значение ошибки результата.

ИПА и ИПД могут оказать влияние на решение задачи при выполнении команд считывания информации.

Безусловный переход от блока через фрагмент $i$ к очередному блоку обеспечивается скорректированным «условием перехода» за счет ИП.
Таким образом, на рис. 5 представлена ИВС, сформированная с учетом возможной некорректности отдельных элементов составных частей, выраженных в формате ИП. Конкретное содержание блоков и фрагментов ИВС должно соответствовать функциональному назначению задачи.

Из материалов статьи следует, что точность решения задачи зависит от величины отклонения условий перехода от нормы, что аналогично степени структуризации составных частей ИВС.

\section{Заключение}

1. Разработана технология формирования ИВС для решения измерительных задач с различной степенью структуризации.

2. В основу решения проблемы положены принципы структурного анализа, позволяющие значительно упростить процесс решения подобных задач.

3. В качестве примера рассмотрена задача определения параметров движения КА, где показано 


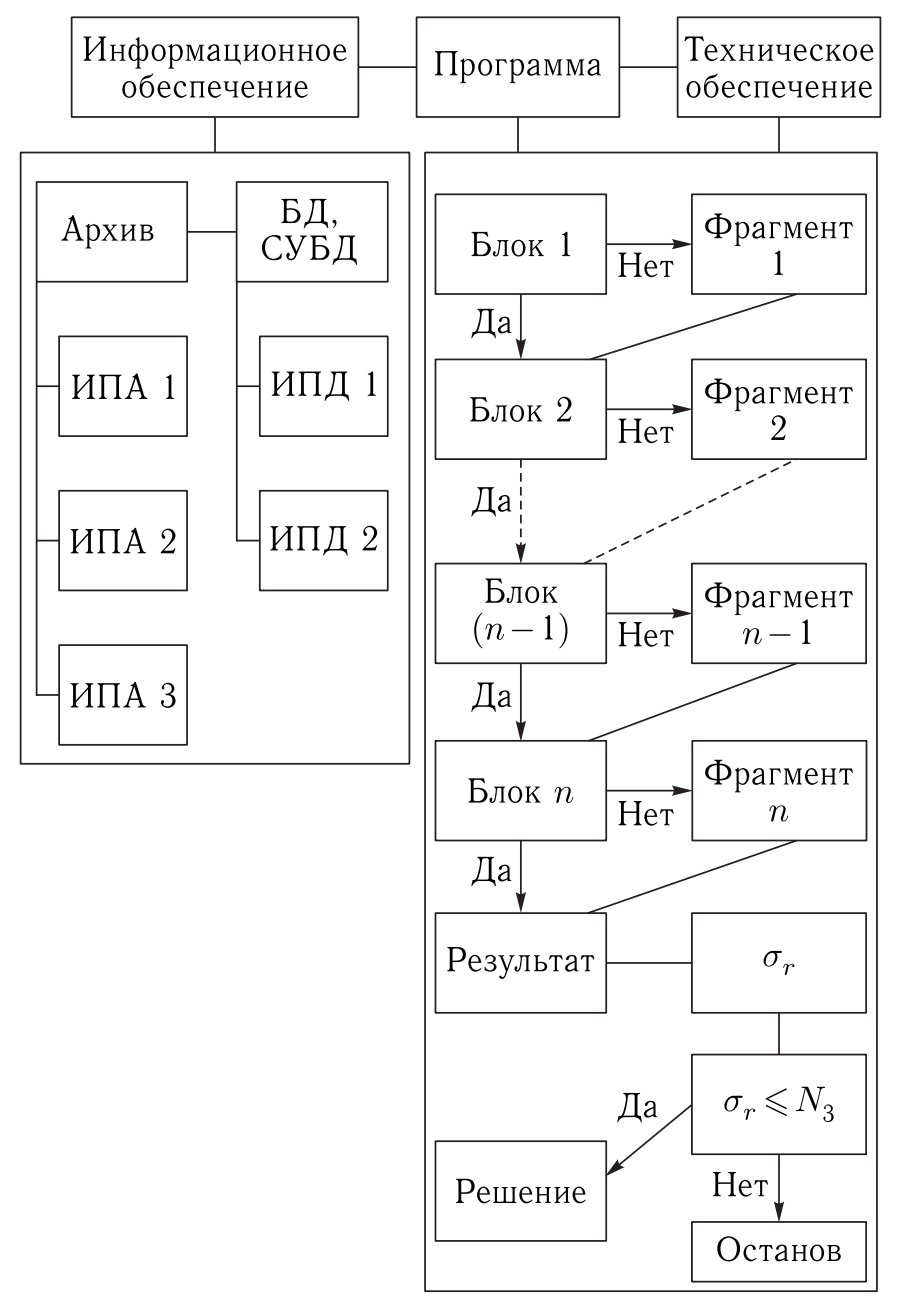

Рис. 5. Структурная схема ИВС

влияние «условий перехода» на точность решения задачи.

4. Предложенная структура ИВС имеет универсальный характер и может использоваться для решения измерительных задач различного типа.

\section{Список литературы}

1. ГОСТ 24453-80 Измерения параметров и характеристик лазерного излучения. Термины, определения и буквенные обозначения величин.

2. ГОСТ Р 53622-2009 Информационные технологии. Информационно-вычислительные системы. Стадии и этапы жизненного цикла, виды и комплектность документов.

3. Йордан Э. Современный структурный анализ. Йордан Пресс Computing Series, 1989. ISBN 0-13598624-9.

4. Рассел С., Норвиг П. Искусственный интеллект: современный подход. 2-е изд. М.: Вильямс, 2007. 1408 с.

5. Бетанов В. В., Ларин В.К. Использование системного подхода к решению проблемных вопросов функционирования АКП БНО полетов КА ГНСС // Ракетно-космическое приборостроение и информационные системы, 2016, т. 3, вып. 1. С. 3-10.

6. Бетанов В.В., Ларин В.К. Концепция построения технологической модели решения слабоструктурированных задач на основе теории множеств // Ракетнокосмическое приборостроение и информационные системы, 2019, т. 6, вып. 2. С. 59-67.

7. Ларин В.К. Построение прототипа экспертно-диагностической системы анализа траекторной измерительной информации // Ракетно-космическое приборостроение и информационные системы, 2017, т. 4, вып. 1. С. 53-60.

8. Бетанов В.В., Ларин В.К., Позяева З.А. К вопросу анализа причин возникновения сбоев в аппаратнопрограммном комплексе уточнения эфемеридно-временной информации ГНСС // Ракетно-космическое приборостроение и информационные системы, 2014, т. 1, вып. 1. С. 55-60. 